\title{
¿Cómo se convierte un diccionario general en un diccionario de ELE?
}

\author{
JIANI LIU \\ Universitat Autònoma de Barcelona \\ jiani.liu@autonoma.cat
}

\begin{abstract}
Resumen
Tradicionalmente se ha considerado que los diccionarios didácticos eran reducciones de los diccionarios generales: alrededor de un diccionario general, proliferaba una familia de diccionarios particulares, entre los que no faltaba uno dirigido a usuarios que estaban en un proceso de aprendizaje de la lengua. Sin embargo, con la evolución de la lexicografía de un tiempo a esta parte, esas soluciones han dejado de ser satisfactorias, ya que se espera que los diccionarios respondan a las necesidades específicas de cada tipo de usuario. En el español tenemos el caso de dos diccionarios de gran prestigio de la editorial SM, el Clave, diccionario general, y el Diccionario de español para extranjeros, entre los que se identifica una clara relación. Con el fin de hacer explícitos los criterios que se aplican a la hora de elaborar un diccionario de aprendizaje a partir de uno general, se realiza un estudio comparativo a partir de un corpus del $5 \%$ de las voces de los dos volúmenes para demostrar que, en este caso, el diccionario de español para extranjeros no es solo una reducción del diccionario general, sino que se han introducido modificaciones pensadas específicamente para los aprendices de segunda lengua o lenguas extranjeras tanto en la macroestructura como en la microestructura.
\end{abstract}

\section{Palabras clave}

Lexicografía pedagógica; diccionario monolingüe; ELE; diccionario de español para extranjeros. 


\title{
How to convert a general dictionary into an SFL dictionary?
}

\begin{abstract}
Traditionally, didactic dictionaries were considered as the simplifications of general dictionaries. A series of more targeted dictionaries can be derived from a general dictionary, among which there was no lack of one for users who were in a process of learning the language. However, with the evolution of lexicography, these solutions are no longer satisfactory, since dictionaries are expected to respond to the specific needs of each type of user. For Spanish, there are two highly prestigious dictionaries from the SM publishing house, the Clave, which is a general dictionary, and the Dictionary of Spanish for Foreigners, between which a clear relationship has been identified. In order to figure out the criteria that is applied when compiling a learning dictionary from a general one, a comparative study is conducted from the $5 \%$ voice corpus of the two volumes. The statistic results demonstrate that the Spanish dictionary for foreigners is not only the simplification of the general dictionary, but also modified for second language learners specifically in both the macrostructure and microstructure.
\end{abstract}

\section{Keywords}

Pedagogical lexicography; monolingual dictionary; SFL; dictionary of Spanish for foreigners.

Recibido el 28/03/2021

Aceptado el 04/11/2021 


\section{Introducción}

A pesar de que toda obra lexicográfica cumple con una función pedagógica, los diccionarios de aprendizaje difieren de los generales por su propósito de atender las necesidades que surgen en el proceso de aprendizaje de una lengua. Teniendo en cuenta el perfil de los usuarios, las obras lexicográficas didácticas se pueden clasificar en dos tipos: diccionarios escolares y diccionarios de aprendizaje para extranjeros (Azorín Fernández, 2000; Humberto Hernández, 2008 y 2015; Porto Dapena, 2002; Perdiguero, 2010), puesto que no son iguales las necesidades de un nativo que está perfeccionando su lengua materna y las de un aprendiz de otro idioma que está aprendiendo una lengua extranjera. De acuerdo con Tarp (2011: 222), «first-language learners are developing their conceptual thinking and knowledge of the world together with their language skills, whereas second-language learners have already developed their conceptual thinking and knowledge beyond their second-language skills».

El origen de la lexicografía española para extranjeros se puede remontar al siglo $X X$, cuando se empieza a comprender la importancia de la enseñanza de español como lengua extranjera. En comparación con el mundo anglosajón, el despegue del ámbito hispánico ha tardado medio siglo en producirse, pero el número de diccionarios de aprendizaje publicados hasta hoy en tan poco tiempo comienza a ser significativo (Nomdedeu, 2006: 403). En 1995 apareció el primer diccionario de aprendizaje de español, el Diccionario para la enseñanza de la lengua española [DIPELE], seguido por el Diccionario Salamanca de la lengua española [SALAMANCA], publicado un año más tarde. En el año 2002 se elaboraron dos repertorios más, el Diccionario de español para extranjeros [DELE] y el Diccionario de la lengua española para estudiantes de español [ESPASA].

En la tradición española, la lexicografía monolingüe del español para usuarios extranjeros se ha abordado principalmente desde dos perspectivas: por un lado, existen trabajos dedicados a la teoría, que incluye la aparición, el desarrollo y la delimitación de los diccionarios de aprendizaje (Humberto Hernández, 1990, 1993, 1996, 2000, 2008; Ciro, 2007) y, por el otro, contamos con estudios que analizan los diccionarios publicados de ELE o alguna parcela de la microestructura de estos diccionarios (González García, 2004; Molina Díaz, 2010; Ruhstaller, 2010; Gordón, 2010; Maldonado González, 2012; Nomdedeu, 2006, 2011). Además, también se han realizado diversas investigaciones empíricas en las que se encuesta a los usuarios extranjeros con el fin de conocer sus hábitos de consulta (Humberto Hernández, 2005; Azorín Fernández y Martínez, 2010; Nomdedeu, 2019). 
En lo que concierne a los rasgos que caracterizan un diccionario monolingüe para extranjeros, lexicógrafos como Humberto Hernández (1990: 164), Azorín Fernández (2000: 22) y Moreno Fernández (2000: 153) aportan, respectivamente, sus consideraciones y, según el último, las características de un diccionario de aprendizaje se sintetizan en cuatro puntos: selección adecuada del léxico, definiciones que aseguren la comprensión, ejemplos para ilustrar las definiciones e información sobre el uso.

Tal como afirma Garriga (1999: 43), en muchas ocasiones, la elaboración de un diccionario más específico se realiza mediante unos procedimientos de reducción de un diccionario general, con el fin de adecuarse, especialmente, a los usuarios escolares y extranjeros. Cabe destacar que es un fenómeno propio de la lexicografía española y el procedimiento de elaborar diccionarios específicos sobre la base de datos de los diccionarios generales también se aplica a la lexicografía digital. No obstante, todavía no contamos con un diccionario nativo digital dirigido extranjeros, razón por la cual este estudio se centra en el análisis comparativo entre dos diccionarios impresos.

Este artículo tiene como objeto de investigación el diccionario Clave, Diccionario de uso del español actual, de carácter general, y el Diccionario de español para extranjeros, una obra lexicográfica didáctica de ELE. Publicados por la misma editorial, SM, el primero recopila una gran cantidad de léxico del español actual, así como americanismos, locuciones e información fonológica, gramatical y de uso, mientras que el segundo se caracteriza por recoger el léxico del llamado «español global», que, como se dice el prólogo, «abarca el español de América Latina y de España» (DELE, 2002: 7) y en el cual se realizan muchos ajustes con el fin de atender las necesidades de los extranjeros.

El presente artículo pretende, en primer lugar, hacer explícitos los criterios que se siguen en el proceso de transformación de un diccionario general en uno didáctico. Pondré el foco en las diferencias entre Clave y DELE a fin de mostrar las modificaciones que se introducen para convertir un diccionario monolingüe para nativos en una obra lexicográfica para extranjeros. Además, trataré de destacar los aspectos positivos del DELE y algunos otros en los que introducir mejoras. Partiendo de este análisis, espero determinar si el Diccionario para extranjeros es adecuado para el objetivo de favorecer el aprendizaje de español por parte de los estudiantes no nativos.

Para el estudio, he constituido un corpus que contiene un $5 \%$ de las palabras de Clave. A fin de realizar el análisis comparativo entre las dos obras lexicográficas, he tomado Clave como referencia, ya que, al ser un diccionario general, su nomenclatura es más amplia que la del DELE. 
Para el análisis, seguiré el esquema que utiliza Garriga (2002: 37) para describir los aspectos más importantes de un diccionario:

Tabla 1. Esquema descriptivo de los aspectos del diccionario ${ }^{1}$

\begin{tabular}{|c|c|c|}
\hline \multirow{2}{*}{$\begin{array}{c}\text { CARACTERÍSTICAS } \\
\text { EXTERNAS }\end{array}$} & \multicolumn{2}{|c|}{ CARACTERÍSTICAS TÉCNICAS } \\
\hline & Macroestructura & Microestructura \\
\hline $\begin{array}{l}\text { - Formato y } \\
\text { encuadernación } \\
\text { - Título e } \\
\text { informaciones } \\
\text { externas } \\
\text { - Editorial } \\
\text { - Responsables de la } \\
\text { obra } \\
\text { - Presentación } \\
\text { - Tipografía }\end{array}$ & $\begin{array}{l}\text { - Número de voces } \\
\text { - Criterios de selección } \\
\text { - Lematización } \\
\text { - Ilustraciones }\end{array}$ & $\begin{array}{l}\text { - Definición } \\
\text { - Acepciones } \\
\text { - Ejemplos } \\
\text { - Información gramatical } \\
\text { - Información sobre el uso } \\
\text { - Expresiones } \\
\text { - Sinónimos y antónimos } \\
\text { - Pronunciación } \\
\text { - Etimología } \\
\text { - Apéndices }\end{array}$ \\
\hline
\end{tabular}

\section{Características externas}

La primera cuestión que llama la atención es la dimensión de los equipos de lexicógrafos de cada diccionario. En el caso de Clave, el equipo de redacción está compuesto por 16 expertos, mientras que solo 7 lexicógrafos se encargan de la redacción de DELE. Aparte, 26 expertos toman la responsabilidad de revisión científica de Clave, pero el equipo de revisión de DELE está formado por solo 5 lexicógrafos. Es razonable que la elaboración de un diccionario monolingüe general sea un trabajo más complejo, y que, por eso, se necesite la cooperación de más lexicógrafos. En lo que se refiere a los paratextos, Clave compara la norma castellana y la meridional, que no se menciona en $D E L E$, ya que dar a conocer estas normas no es uno de los objetivos principales de un diccionario didáctico para extranjeros. Además, el alfabeto fonético que DELE dispone en las primeras páginas es de mucha utilidad para los alumnos de español como segunda lengua o como lengua extranjera, especialmente para los principiantes, dado que la transcripción fonética de los lemas es una de las informaciones que proporciona.

${ }^{1}$ En el anexo 1 se presenta una muestra del corpus. 


\section{Macroestructura}

\subsection{Selección de voces}

En el $5 \%$ de la muestra que he seleccionado, Clave registra 2.045 voces y DELE, 1.459. Si se hace la proyección a la totalidad de ambos diccionarios, resulta que en Clave se recopilan, aproximadamente 40.900 lemas, mientras que DELE incluye 29.180. Comparando el número de voces de las dos obras lexicográficas, el vocabulario de DELE solo ocuparía un $71 \%$ del de Clave. Dicho de otra manera, DELE perdería el 29\% de la nomenclatura de Clave.

Con el fin de revelar las diferencias acerca de los criterios de selección de léxico entre ambas obras, he realizado el análisis de las 619 voces del corpus que pierde el $D E L E$ y las 33 palabras que incorpora respecto al Clave. Los lemas que solo se encuentran en uno de los dos diccionarios se pueden clasificar en los siguientes grupos: siglas, formas verbales irregulares, palabras derivadas, préstamos, palabras formadas con prefijos y sufijos y términos especializados.

El apartado de «Características del diccionario» de DELE (2002: 9) señala que se recogen las siglas y abreviaturas. En el corpus, se observan cinco siglas (AA.VV., BOE, $D N I, O T I, T V E$ ) que solo aparecen en DELE. Considerando que los aprendices de ELE pueden tener conocimientos muy limitados acerca de las siglas y abreviaturas del español, es necesario recopilarlas en el diccionario. Este criterio se ve reflejado en las palabras de Castillo Carballo (2003: 95), quien afirma que ya es una tendencia reciente la inclusión de las siglas y abreviaturas en los diccionarios.

El DELE avisa de que «para facilitar su búsqueda en el diccionario, se han incluido las formas verbales irregulares que no se identifican claramente con su infinitivo» (DELE, 2002: 9). Por ejemplo, en DELE se pueden encontrar artículos como: diga, eres, pida, plazca, rayáis, riáis, sois, tañendo, tañera, tañó, tuve, zurza, ninguno los cuales se registra en Clave. Estos 12 casos representan un 36\% de las voces propias de la muestra recogida en DELE. El registro de las formas irregulares verbales como entradas independientes es un aspecto muy favorable para los aprendices de español, sobre todo para las personas cuya lengua materna no tiene flexión.

En Clave, las palabras derivadas se registran como entrada, pero, en el caso de $D E L E$, en muchas ocasiones se sitúan al final del artículo de la palabra que origina la derivación. Por ejemplo, la palabra adhesividad es un lema en Clave, pero en DELE, aparece dentro del artículo de adhesivo, presentada como: ${ }^{\circ} F A M$. adhesividad. Teniéndose en cuenta que $D E L E$ es un diccionario didáctico para extranjeros concebido en soporte papel, es lógico que racionalice el espacio y no presente todas las palabras derivadas de manera detallada para, en algunas ocasiones, evitar la 
repetición de definiciones parecidas. No obstante, tratándose de un diccionario para no nativos, no se puede suponer que los usuarios conozcan las reglas de formación de palabras hasta el punto de deducir el significado de un derivado a partir de la palabra de la que deriva, ni la categoría gramatical a la que pertenecen. De hecho, los estudiantes no son capaces de poner estas palabras en práctica con solo conocer su significado.

El diccionario Clave ofrece algunos préstamos de uso frecuente en el mundo hispánico, 22 de los cuales no se registran en DELE; en otras palabras, un 3,6\% de las voces que pierde DELE son préstamos, por ejemplo: extranjerismos: calvados (fr.), cascadeur (fr.), dixie (ing.), graben (al.), jumper (ing.), laptop (ing.), mujik (rus.), nurse (ing.), pidgin (ing.), portfolio (ing.), prime rate (ing.), rating (ing.), soirée (fr.), twinset (ing.), tweed (ing.), vichyssoise (fr.), vichy (fr.); latinismos: ab absurdo (lat.), ab aeterno (lat.), ad interim (lat.), corpus delicti (lat.), criterium (lat.). En algunos casos, el uso de los préstamos no es necesario, ya que se puede sustituir con léxico propio del español. Así pues, en un diccionario para el aprendizaje de español como lengua extranjera es lógico que se limite la inclusión de este tipo de voces.

Clave y DELE muestran una disparidad relevante con respecto al registro de algunas palabras formadas con prefijos y sufijos: el Clave las trata como entradas, mientras que el DELE las suprime. Son ejemplos de ello: desenlazar; inadecuado, da; inactual; inagotable; insurreccionar; microestructura; neoplatonismo, etc. En el corpus de estudio han aparecido 149 palabras prefijadas que se eliminan del $D E L E$, lo que supone un $24 \%$ del total de palabras suprimidas. En realidad, gracias a la productividad de los prefijos y sufijos, el hablante puede formar numerosas palabras nuevas, la gran mayoría de las cuales se usa con mucha frecuencia en la comunicación cotidiana, así que los diccionarios didácticos para extranjeros también deberían incluir estos términos, máxime cuando se trata de usuarios cuyo conocimiento de la creatividad léxica del español es limitado.

Aparte de lo comentado en los párrafos anteriores, he observado también que Clave abarca muchos términos de nombres especializados, o relacionados con ellos, de distintas ciencias y técnicas (botánica, zoología, física, química, medicina, religión, etc.) que no se pueden encontrar en DELE. En total en el corpus se observan 96 palabras que suprime el $D E L E$, lo que supone un $15,5 \%$ de todas las palabras suprimidas en este diccionario. En virtud de que la demanda primordial de los aprendices extranjeros cuando consultan un diccionario es la de resolver problemas lingüísticos acerca del vocabulario cotidiano, estas voces de nombres específicos no son tan necesarias para ellos, ya que en pocas ocasiones tienen oportunidad de usarlas. 
En fin, el distinto perfil de los usuarios de los dos diccionarios es la causa de estos aspectos diferentes en los criterios selectivos, porque, tal y como señala Porto Dapena (2002: 171), la selección del léxico de un diccionario depende, en cierto sentido, de su finalidad, o sea, de sus destinatarios.

\subsection{Lematización}

La comparación entre cómo se lematiza en Clave y DELE muestra tres aspectos en los que difieren. Como se indica en su cubierta posterior y en la parte «Ejemplos de uso», una de las peculiaridades de DELE consiste en la separación silábica, diseñada «para saber cómo se separa una palabra al final de línea y para acentuar correctamente» (DELE, 2002: 12). Beneficia, en cierto sentido, a los estudiantes de español de nivel inicial, aunque podría obstaculizar la consulta a los estudiantes con un nivel medio o alto.

La segunda característica propia de Clave, que lo diferencia de otros diccionarios, y también de DELE, es el uso de corchetes iniciales cuando un lema o acepción no está registrado en la vigésima edición del Diccionario de la RAE (Clave, 1999: XV), por ejemplo: [antropozoico, ca, [barraquismo, [gulag, [paparazzi, [software, etc. En realidad, el uso propio del corchete inicial de Clave se puede considerar como un diseño que pierde su sentido, porque el Diccionario de la Lengua Española se actualiza periódicamente, y algunas palabras marcadas con corchete en Clave ya se han registrado en la última edición de $D L E$ o en alguna de sus actualizaciones, por ejemplo, container, digitalización, sufijar, etc.

Otra diferencia consiste en la lematización de las palabras terminadas en consonante y las que tienen flexión de género: realizador, -a (Clave), realizador, dora (DELE); madrugador, -a (Clave), madrugador, dora (DELE); portugués, -a (Clave), portugués, guesa (DELE); pastor, -a (Clave), pastor, tora (DELE); ratón, -a, (Clave), ratón, tona (DELE); siamés, - a (Clave), siamés, mesa (DELE), etc. La estrategia de DELE de proporcionar la terminación de la palabra desde la sílaba tónica aporta más claridad y reduce la posibilidad de confusión en relación a la pérdida de la tilde en el plural en determinados casos.

\subsection{Ilustraciones}

En Clave no hay ilustraciones, ni siquiera en los apéndices. En cambio, DELE contiene 26 ilustraciones dentro del texto en todo el diccionario, sin contar las que están en los apéndices. Los artículos de la muestra acompañados con imágenes son: abanico, acueducto, baraja, boleadoras, bota, botijo, castañuelas, cataratas, cocinar, fregona, futbolín, gaita, guitarra, hoja, hórreo, llama, mantón, mariachi, molino, paella, 
poncho, porrón, quena, rebozo, vieira, zambomba. Parece que la razón por la que $D E L E$ recoge ilustraciones que acompañan a algunas palabras se debe a que estas voces disponen de un valor cultural, que es propio de España, o del mundo hispánico. Como vio Garriga (2002: 42), «[...] un diccionario de la lengua no tiene por qué contener ilustraciones; incluso se considera que pueden ser confusas desde el punto de vista teórico, al identificar el significado de un signo lingüístico con el objeto. Por eso es común que los grandes diccionarios no contengan ilustraciones, a diferencia de las enciclopedias, donde son características». No obstante, hay que reconocer que las ilustraciones pueden desempeñar un rol importante en un diccionario, ayudando a la codificación, mejorando la comprensión de determinadas definiciones, en especial las que tienen que ver con la ciencia y la técnica (Rodríguez y Garriga, 2016). De acuerdo con Rodríguez (2012: 172-173), la ilustración constituye un atractivo elemento semiológico que adquiere gran protagonismo en aquellos diccionarios que aspiran a un fin didáctico en el conocimiento y aprendizaje de una lengua.

\section{Microestructura}

\subsection{Definición}

Según el corpus que he elaborado, de 1.426 palabras que coinciden en los dos diccionarios, solo en 79 de ellas se observan diferencias en cuanto a la definición, es decir, solo un 5,5\% de las definiciones introducen alguna variación. Dicho de otra manera, el 94,5\% de las definiciones de la muestra coincide exactamente entre ambos diccionarios. Las modificaciones mínimas se realizan a través de adición o supresión de algunas palabras o la alteración del orden de los elementos de la frase definitoria. La alta coincidencia de las palabras definidoras y las pocas alteraciones introducidas son, en realidad, un rasgo inesperado para un diccionario monolingüe para extranjeros.

En comparación con lo que hace Clave, DELE define los términos especializados en frases más cortas y sencillas. Como vio Medina Guerra (2003: 135), a veces la sencillez no es compatible con la claridad y precisión, especialmente en las definiciones de los tecnicismos o de nombres de plantas y animales. Sin embargo, un estudiante extranjero no necesita tanta información enciclopédica de los elementos químicos, o de otros campos específicos; por tanto, una presentación breve ya es suficiente. Acerca de las definiciones de las siglas, desde el punto de vista de los aprendices extranjeros, la forma de definir las siglas de DELE responde mejor su demanda de consulta, al presentar solo la forma completa de la sigla. Se observa que $D E L E$ selecciona para sus definiciones palabras más usuales y sencillas, que no van a 
necesitar de una segunda búsqueda por parte de los alumnos no nativos, lo cual constituye un criterio muy importante en la redacción de un diccionario didáctico para extranjeros. Véase el siguiente ejemplo:

chapucería 1 Tosquedad, imperfección o deficiente acabado en lo que se hace. [Clave]

chapucería 1 Imperfección o falta de precisión y cuidado. [DELE]

Por lo que respecta a las definiciones de mayor extensión en Clave, pese a que una definición más detallada es en principio mejor, la simplificación de DELE no debería de causar ningún malentendido. Según Nomdedeu (2006: 420), las definiciones en los diccionarios monolingües para extranjeros deben ser claras, concisas y exactas y una estrategia muy útil es la creación de una lista cerrada de definidores, llevada a cabo por primera vez en el español por el DIPELE. Humberto Hernández (2000), Moreno Fernández (2000), González García (2004), Maldonado González (2019) y Sánchez López (2020) también recomiendan la redacción a partir de un reducido número de definidores (2.000 o 3.000 unidades).

\subsection{Acepciones}

De las 1.426 voces que se registran en ambos diccionarios, solo 38 de ellas muestran diferencias acerca del número de acepciones. Es decir, un 97,3\% de las entradas recogidas en ambos volúmenes tiene el mismo número de acepciones. La excesiva dependencia de DELE respecto a Clave afecta en gran medida a la calidad del primero. Al observar las acepciones que pierde el DELE respecto al Clave, una parte de ellas son locuciones. Existen también acepciones propias de Clave marcadas con un corchete inicial. Considerando su ausencia en el diccionario académico, DELE opta por reservar las acepciones de uso más frecuente y eliminar las que no son ampliamente aceptables. Se observa que DELE también suprime algunas acepciones que disponen de un significado especial en determinado campo o situación, por ejemplo:

primo, ma s.f. 5 En la iglesia católica, tercera de las horas canónicas. (Clave)

vuelto, ta s.m. 2 En zonas del español meridional, vuelta o dinero que sobra de pagar algo: Pedí el vuelto al mesero. (Clave)

Es verdad que estas acepciones son interesantes para ampliar el conocimiento, pero en un diccionario para extranjeros, este objetivo desempeña un papel secundario. 


\subsection{Ejemplos}

Según el recuento de los ejemplos registrados en el corpus, Clave incluye 3.389 acepciones y 3.617 ejemplos, con una proporción de 1,07 ejemplos por acepción, mientras que $D E L E$ registra 2.664 acepciones y 3.067 ejemplos y la proporción resultante es de 1,15 ejemplos por acepción.

Cabe destacar que en la muestra de Clave se observan 136 acepciones carentes de ejemplo. En DELE 104 acepciones han aparecido sin ejemplo, lo cual supone un 4\% de todas las acepciones. A pesar de que la proporción de la ausencia del ejemplo no es alta, la supresión de este elemento lexicográfico en un diccionario de aprendizaje para extranjeros es algo que no debería suceder. Tal como señala Prado Aragonés (2004: 159), es imprescindible que cada una de las acepciones vaya acompañada de ejemplos, que servirán para aclarar el significado, mostrar el comportamiento sintáctico de la palabra e ilustrar determinadas combinaciones léxicas frecuentes que no hayan podido ser especificadas en la definición.

Entre las 1.426 entradas que se incluyen en ambos volúmenes, el 49,9\% de las voces del corpus presenta diferencias en los ejemplos. En Clave se utiliza principalmente la frase como unidad para los ejemplos, pero DELE incluye muchos ejemplos truncados, como se puede observar en el siguiente caso:

desafío s.m. 1 Incitación o invitación a la lucha o a la competición: Acepté su desafío porque sé que voy a ganar. 2 Rivalidad o competencia: Estamos preparados para el desafío tecnológico de los próximos años. (Clave)

desafío s.m. 1 Incitación o invitación a la lucha o a la competición: aceptar un desafío. 2 Rivalidad o competencia: el desafío tecnológico. (DELE)

Otra diferencia notable consiste en la longitud de los ejemplos. El Clave prefiere construir ejemplos largos, pero DELE opta por registrar ejemplos breves:

plato s.m. Recipiente bajo, generalmente redondo, con una concavidad central más o menos honda, que se usa para servir las comidas: He fregado los platos y los he colocado en el escurridor. (Clave)

plato s.m. Recipiente bajo, generalmente redondo, con una concavidad central más o menos honda, que se usa para servir las comidas: He fregado los platos. (DELE)

taoísta s. 2 Persona que tiene como religión el taoísmo: Los taoístas aspiran a alcanzar el éxtasis gracias a la meditación, la contemplación y la supresión de todo deseo. (Clave)

taoísta s. 2 Persona que tiene como religión el taoísmo: Los taoístas aspiran a alcanzar el éxtasis gracias a la meditación. (DELE) 
En definitiva, estos ejemplos de DELE experimentan una simplificación de los ejemplos de Clave, lo cual pone en evidencia la inclinación del DELE por ejemplos más concisos y condensados. El ejemplo lexicográfico, como modelo de uso, complemento de la definición, depósito de la información gramatical y cultural, desempeña un papel importante en una obra lexicográfica (Garriga, 2002), y, para un diccionario de aprendizaje, el ejemplo debe ser breve e incluir la máxima información en el menor espacio posible (Hermanns, 1988; Jacinto García, 2015).

\subsection{Marcas de uso y de materia}

En lo referente a las marcas de uso (denominadas como registros de uso en ambos diccionarios), no existe diferencia alguna; en cambio, en el caso de las marcas de materia, sí la hay. El diccionario DELE especifica, con las marcas de materia, los campos en los que se utiliza la palabra entrada. Se representan mediante abreviatura, con todas las letras en mayúscula. Sin embargo, estas marcas en Clave se presentan en forma completa, no en abreviatura. Véanse algunos ejemplos:

antropozoico, ca adj. En geología, de la era cuaternaria, quinta de la historia de la Tierra, o relacionado con ella. (Clave)

antropozoico, ca adj. GEOL. De la era cuaternaria, quinta de la historia de la Tierra, o relacionado con ella. (DELE)

defectivo, va adj. En gramática, referido a un verbo, que no se usa en todas las formas de su conjugación. (Clave)

defectivo, va adj. GRAM. Referido a un verbo, que no se usa en todas las formas de su conjugación. (DELE)

En realidad, los estudiantes de un idioma extranjero siempre se enfrentan con la dificultad de interpretar abreviaturas por la limitación de su vocabulario, por eso esta información previa a la definición en DELE podría ser ignorada por ellos. Sin embargo, cuando una palabra tiene varias acepciones, puede que solo en determinados campos adquiera algún significado especial, de modo que dicha información juega un papel imprescindible. Por eso, es conveniente que se presenten de manera más completa, como lo hace Clave.

\subsection{Sinónimos y antónimos}

La existencia de sinónimos y antónimos hace posible la relación interna de las entradas de un diccionario y ayuda a los aprendientes no nativos a comprender y usar el vocabulario. Según se apunta en la información previa de cada diccionario, en los artículos de términos sinónimos se repite la definición y se añade el sinónimo antes 
del ejemplo (Clave, 1999: XVII), mientras que DELE registra los principales sinónimos y también los antónimos del español actual y especifica si se refieren a una acepción determinada o a toda la palabra (DELE, 2002: 10). No obstante, de acuerdo con el recuento del corpus, el dato de tipo paradigmático de los dos diccionarios es escaso: 235 (11\%) en el caso de Clave y 193 (13\%) en DEPE.

\subsection{Locuciones}

La desigualdad del registro de las locuciones entre los dos diccionarios objeto de estudio consiste en el formato. En DELE las locuciones se recogen en una acepción aparte, normalmente la última, pero el Clave incluye las locuciones en las acepciones correspondientes. Otra diferencia estriba en que el DELE sustituye el lema-entrada (si no tiene flexión genérica) con una virgulilla, mientras Clave repite la palabra entrada en todas las locuciones, por ejemplo:

$$
\begin{aligned}
& \text { más } 5 \text { || a más no poder; [...] || a más y mejor; [...]. (Clave) } \\
& \text { más } 5 \text { || a no poder; [...] || a y mejor; [...]. (DELE) }
\end{aligned}
$$

Como se puede observar, la forma de presentar las locuciones de Clave es más completa y clara, pero DELE utiliza este método en beneficio de la brevedad y el ahorro de espacio. Aun así, pensando en los formatos electrónicos, no hay que despreciar la redundancia.

\subsection{Etimologías}

La eliminación de la información sobre etimología en DELE es una modificación que merece discusión. Según afirma Garriga (2002: 47), en una tradición como la de la lexicografía española es frecuente que se proporcione la etimología de las palabras, sin embargo, parece evidente que para usar correctamente una palabra no se requiere conocer su étimo. Moreno Fernández (2000: 154) también opina que los diccionarios para extranjeros deberían prescindir de la información histórica o etimológica. Sin embargo, para un estudiante francés, portugués o italiano avanzado de español puede resultar útil conocer el origen latino de algunas palabras para poderlas relacionar más fácilmente con las de su lengua materna.

\subsection{Pronunciación}

De acuerdo con el recuento de los datos, el Clave proporciona la información sobre la pronunciación de 84 extranjerismos y, por parte de DELE, el número de extranjerismos acompañados con la transcripción fonética es de 43. Cabe enfatizar que esta información resulta de provecho para un mejor dominio de los 
extranjerismos porque, al introducirse en la lengua española, los préstamos experimentan cierto grado de adaptación fonética, así que tanto los nativos como los alumnos extranjeros necesitan saber cómo se pronuncia; así lo indican los diccionarios en casos como megabyte ${ }^{\square}$ Pron. [megabáit], playboy ${ }^{\square}$ Pron. [pléiboi], etc. No obstante, para los aprendientes de nivel inicial o intermedio será útil que el diccionario proporcione la transcripción fonética de todas las palabras (Azorín Fernández y Martínez Egido, 2010), como lo observado en el DIPELE y el ESPASA.

\subsection{Información gramatical y de uso}

Entre las 1.465 entradas de la muestra que aparecen en ambos diccionarios, se han observado en Clave 118 voces con la información ortográfica y, por parte de DELE, el dato es de 90. Las informaciones ortográficas que coinciden en Clave y DELE se pueden clasificar, principalmente, en dos categorías: notas sobre el cambio de letras de algunas palabras cuando se conjugan (por ejemplo, despojar ${ }^{\square}$ Conserva la $j$ en toda la conjugación) y notas que explican si alguna letra de cierta palabra lleva o no la tilde cuando flexiona (por ejemplo, contagiar $^{\square}$ La $i$ nunca lleva tilde).

En lo que concierne a la inclusión de la información morfológica, 164 voces de la muestra de Clave ofrecen dichas indicaciones, mientras que el número de artículos que contienen la información morfológica de DELE es de 344. Aparte de la indicación de la manera de conjugar los verbos irregulares y la forma plural o epicena de los sustantivos, DELE también proporciona dicha información para los verbos regulares o los sustantivos cuya terminación lleva tilde. Estos dos diseños de DELE favorecen en gran medida a los alumnos de lenguas extranjeras, porque la conjugación verbal y el plural de los sustantivos constituyen dos problemas frecuentes en la fase inicial de su aprendizaje.

La información semántica incluida en los dos diccionarios es muy escasa, pues en DELE solo se observan 10 artículos registrados con este tipo de información. En Clave se incluyen 59 casos más, que sirven para evitar confusión y señalar matizaciones diferenciadoras ${ }^{2}$, lo que responde más a las demandas de usuarios con un dominio avanzado de español. Por tanto, sin conocer esta información, los extranjeros tampoco van a resolver algunas de las dificultades que les plantee el uso.

En DELE solo se encuentran 35 artículos en los que se incluye la información sintáctica y Clave registra 6 más. Las notas de sintaxis que se incluyen en los dos volúmenes indican la construcción verbal o el uso incorrecto de alguna combinación.

2 Por ejemplo: bibliográfico, ca $\square$ SEM. Dist. de biográfico (de la vida de una persona). 
Dicha información es muy apropiada para los estudiantes extranjeros porque el conocimiento de las palabras aisladas no es suficiente para el uso de un idioma.

Clave anuncia el uso innecesario de algunos anglicismos en 29 artículos lexicográficos, información que no está incluida en DELE. Sin ella, un alumno no nativo podría considerar un anglicismo como la única forma de expresar un significado. Se han observado 28 casos en DELE que cuentan con información sobre el uso del lema, y varios de ellos indican el uso de algunos americanismos en España o Canarias, lo cual sirve para recoger palabras de las diferentes variedades de español. Por parte de los estudiantes extranjeros, también conviene conocer el uso frecuente de determinados términos típicos del español atlántico.

\subsection{Apéndices}

Los apéndices de Clave tienen carácter global y sistemático, abarcando «Acentuación», «Puntuación», «División de palabras», «Uso de las mayúsculas», «Escritura de números», «Abreviaturas y símbolos», «Siglas y acrónimos», «Topónimos» y «Modelos de conjugación verbal». En cambio, los apéndices de DELE están compuestos por dos apartados: «Guía de conversación» $\mathrm{y}$ «El verbo. Modelos de conjugación». En el primero se diseñan diversas situaciones comunicativas reales y el segundo sirve como referencia a las notas morfológicas que se integran en la microestructura. La parte de apéndices de Clave tiene un valor didáctico, en la que se explican, de manera precisa y detallada, muchos conocimientos lingüísticos, mientras que los apéndices de $D E L E$ ponen el foco en la aplicación del español en la conversación real, de lo que pueden sacar buen provecho los aprendices. En suma, los apéndices de Clave son más generales y teóricos y, en el caso de DELE, son más prácticos para el uso del idioma.

\section{Conclusión}

Ciertamente, sobre la base de Clave, DELE realiza modificaciones para atender las necesidades de los estudiantes de español como lengua extranjera. A partir del análisis realizado, destacan dos aspectos: la selección de voces y la inclusión del ejemplo lexicográfico. La definición, la lematización, las acepciones, las locuciones, y la información gramatical y de uso y los apéndices presentan algunas diferencias, pero no son muy notables. Son características propias de DELE la inclusión de ilustraciones y antónimos, mientras que Clave, por su parte, ofrece la etimología de las voces. Algunas de mis conclusiones coinciden con los aspectos comunes de los diccionarios didácticos que plantea Azorín Fernández (2000), como son el control del vocabulario y el tipo de ejemplificación. Sin embargo, DELE no presta demasiada atención a la 
inclusión de notas gramaticales y de uso, que son de gran utilidad para el aprendiz extranjero, especialmente cuando tiene problemas relacionados con la producción de textos (Jesen y Tarp, 2019).

A través de la comparación de los dos diccionarios, se puede concluir que las ventajas de DELE son la exhaustiva inclusión de los ejemplos que favorecen la comprensión de los lemas, un lenguaje breve y de fácil acceso en las definiciones, la inclusión de notas morfológicas sobre la conjugación verbal y las formas plurales de los sustantivos, así como la separación silábica, las ilustraciones y la guía de conversación. Estos puntos fuertes de DELE cumplen con algunos de los requisitos que plantea Maldonado González (2012: 174), pero ella misma también reconoce que el $D E L E$ ya no se considera muy competente para atender las necesidades de los aprendientes de español como lengua extranjera:

[...] el análisis de los datos de las consultas diarias realizadas a Clave en línea desde 2001, nos permitió constatar que, de esas casi 500000 visitas mensuales, casi dos tercios correspondían a usuarios extranjeros que estaban aprendiendo español como segunda lengua, dentro y fuera de España. Este análisis cualitativo de los datos cuantitativos explica por qué no se ha relanzado ni reeditado nuestro Diccionario de español para extranjeros, cuya primera edición en papel, de 2002, se convirtió en la única, pese a contar con varias reimpresiones. Como ya quedó reflejado en Maldonado (2012), las cosas cambian, y lo que en 2002 nos pareció un proyecto válido, innovador y avanzado de diccionario monolingüe de español para extranjeros es hoy un planteamiento que no satisface al usuario, en un sector cada vez más especializado por las distintas necesidades de aprendizaje de, pongamos por caso, aprendices sinohablantes frente a aprendices de español de origen italiano o estadounidense. (Maldonado González, 2019: 108-109)

Efectivamente, la alta coincidencia entre Clave y DELE ha mostrado que el segundo resulta ser deudor del primero, sobre todo en la redacción de la definición. Además, cabe destacar que el DELE procura dirigirse a todos los estudiantes extranjeros en general, sin diferenciarlos por el origen, o por nivel lingüístico. No obstante, los principiantes, los aprendientes de nivel intermedio y los de nivel avanzado tienen diferentes niveles a la hora de comprender y producir textos, por eso los diccionarios generales de aprendizaje deben graduarse según el dominio de la lengua extranjera (Tarp, 2006: 313-315).

Otro enfoque que hay que tratar es el papel importante de los diccionarios de aprendizaje en la codificación. Aunque el uso tradicional de las obras lexicográficas consiste en la búsqueda de palabras desconocidas y la consulta de dudas ortográficas, un diccionario, si se concibe realmente como un diccionario didáctico, debe proporcionar información de muy diverso tipo para facilitar el uso de la lengua 
(Maldonado González, 2003: 143). Nomdedeu y Tarp (2018), después de analizar los diccionarios de aprendizaje para extranjeros, concluyen que tanto $D E L E$, como los diccionarios de este tipo que se encuentran en el mercado no han fomentado dicha condición por la carencia de datos gramaticales y la insuficiencia de las informaciones sintácticas, paradigmáticas y culturales.

En definitiva, el análisis contrastivo entre Clave y DELE ha mostrado que le falta mucho a DELE para ser una herramienta adecuada para el aprendizaje de español para extranjeros. Sin embargo, las deficiencias de DELE pueden convertirse en nuestro punto de partida, ya que la lexicografía de aprendizaje se va desarrollando y actualizando gracias a los estudios e investigaciones lexicográficas, así como la aplicación de las nuevas tecnologías de la información y de la comunicación.

\section{Referencias bibliográficas}

AZORÍN FERNÁNDEZ, D. (2000). Los diccionarios didácticos del español desde la perspectiva de sus destinatarios. ELUA. Estudio de Lingüística, N. 14, 19-44.

AZORÍN FERNÁNDEZ, D. y MARTÍNEZ EGIDO, J. J. (2010). Sobre la utilidad de los diccionarios monolingües de aprendizaje (DMA) para estudiantes de ELE. En RUHSTALLER, S y DOLORES GORDÓN, M. (eds.). Diccionario y aprendizaje del español. Suiza. Peter Lang, 89-132.

CASTILLO CARBALLO, M. A. (2003). La macroestructura del diccionario. En GUERRA, A. M. M. (eds.). Lexicografía española. España. Ariel, 79-102.

CIRO, L. A. (2007). El diccionario como objeto de estudio y herramienta didáctica en la enseñanza de la lengua. El Ágora USB, 7(1), 57-64.

GARRIGA ESCRIBANO, C. (1999). Diccionarios didácticos y marcas lexicográficas. En VILA, M. N. (eds.). Así son los diccionarios. Servicio de Publicaciones, 43-76.

GARRIGA ESCRIBANO, C. (2002). Los diccionarios escolares. Textos de Didáctica de la Lengua y de la Literatura, 31, 35-52.

GARRIGA ESCRIBANO, C. (2001). Los ejemplos en los diccionarios didácticos del español. En AYALA CASTRO, M. C. (coord.). Diccionarios y enseñanza. Servicio de Publicaciones, 127-149.

GONZÁLEZ GARCÍA, E. (2004). Algunas observaciones en torno a la lexicografía destinada a la enseñanza del español como segunda lengua. En Actas del XV Congreso Internacional de la ASELE. Sevilla, 405-411.

GORDÓN PERAL, M. D. (2010). La caracterización lexicográfica de voces malsonantes en el diccionario monolingüe de aprendizaje del español. En RUHSTALLER, S y DOLORES GORDÓN, M. (eds.). Diccionario y aprendizaje del español (pp. 321355). Suiza. Peter Lang. 
HERMANNS, F. (1988). Das lexikographische Beispiel: Ein Beitrag zu seiner Theorie. En GISELA HARRAS (ed.). Das Wörterbuch: Artikel und Verweisstrukturen. Düsseldorf. Schwann, 161-195.

HERNÁNDEZ, H. (1990). Hacia un modelo de diccionario monolingüe del español para usuarios extranjeros. Actas del I Congreso Nacional de ASELE. Granada. Universidad, 159-166.

HERNÁNDEZ, H. (1993). De la teoría lexicográfica al uso del diccionario: el diccionario en el aula. En MONTESA PEYDRÓ, S. y A. GARRIDO MORAGA (eds.). Actas del III Congreso Internacional de ASELE. El español como lengua extranjera: De la teoría al aula, Málaga, 10-12 de octubre de 1991. Málaga. Universidad de Málaga, 189200.

HERNÁNDEZ, H. (1996). El nacimiento de la lexicografía monolingüe española para usuarios extranjeros. En Actuales tendencias en la enseñanza del español como lengua extranjera II: actas del VI Congreso Internacional de ASELE: (León 5-7 de octubre de 1995). Servicio de Publicaciones, 203-210.

HERNÁNDEZ, H. (2000). El diccionario en la enseñanza de E.L.E. (Diccionarios de español para extranjeros). En Actas del XXI Congreso Internacional de ASELE, 93103.

HERNÁNDEZ, H. (2005). Quince años después: estudio actual y perspectivas de la lexicografía del español para extranjeros. En Actas del XV Congreso Internacional de ASELE. Universidad de Sevilla, 465-472.

HERNÁNDEZ, H. (2008). Retos de la lexicografía didáctica española. En Actas del II Congreso Internacional de Lexicografía Hispánica.

HERNÁNDEZ, H. (2015). Lexicografía académica y lexicografía didáctica: ¿relaciones excluyentes o conceptos complementarios?. Nueva Revista de Filología Hispánica, 2015, 423-443.

JACINTO GARCÍA, E. (2015). Forma y función del diccionario. Hacia una teoría general del ejemplo lexicográfico. Jaén. Universidad de Jaén.

JENSEN, H. D. y TARP, S. (2019). El tratamiento de adjetivos en diccionarios para aprendices extranjeros de español. Revista Internacional de Lenguas Extranjeras, $10,13-47$.

MALDONADO GONZÁLEZ, C. (2003). La lexicografía didáctica monolingüe en español. En MARTÍN ZORRAQUINO, M. A. y ALIAGA JIMÉNEZ, J. L. (coord.). La lexicografía hispánica en el siglo XXI. Balance y perspectivas. Zaragoza. Sansueña Industrias Gráficas, 129-150.

MALDONADO GONZÁLEZ, C. (2012). Los diccionarios en el mundo ELE: ayer, hoy y mañana (una reflexión desde la propia experiencia). Revista Internacional de Lenguas Extranjeras. Tarragona. Publicacions URV, No 1, 129-150. 
MALDONADO GONZÁLEZ, C. (dir.) (1999). CLAVE. Diccionario de uso del español actual. Madrid. Ediciones SM.

MALDONADO GONZÁLEZ, C. (dir.) (2002). Diccionario de español para extranjeros. Madrid. SM.

MEDINA GUERRA, A. M. (2003). La microestructura del diccionario: la definición. En GUERRA, A. M. M. (eds.). Lexicografía espanyola. España. Ariel, 127-150.

MOLINA DÍAZ, F. (2010). El tratamiento de las colocaciones en los diccionarios monolingües de español L2. En RUHSTALLER, S. y DOLORES GORDÓN, M. (eds.). Diccionario y aprendizaje del español. Suiza. Peter Lang, 171-184.

MORENO FERNÁNDEZ, F. (2000). Diccionarios para el aprendizaje de lenguas extranjeras. En RUSHTALLER, S. y PRADO ARAGONÉS, J. (eds.). Tendencias en la investigación lexicográfica del español. El diccionario como objeto de estudio lingüístico y didáctico. Huelva. Universidad de Huelva, 151-170.

NOMDEDEU RULL, A. (2006). Diccionarios monolingües de aprendizaje de E/LE: características y uso. Annali della Università degli Studi di Napoli "L'Orientale". Napoli. L'Orientale Editrice, 397-431.

NOMDEDEU RULL, A. (2011). «El uso del diccionario en el aula de E/LE: problemas y posibles soluciones». En: Marta Albelda Marco. Foro de Profesores de E/LE, VII.

NOMDEDEU RULL, A. y TARP, S. (2018). Hacia un Modelo de Diccionario en Línea para Aprendices de Español como LE/L2. Journal of Spanish Language Teaching, 5(1), 50-65.

PERDIGUERO, V. H. (2010). Logros y retos de los diccionarios de español para extranjeros: noticias halagüeñas. En RUHSTALLER, S y DOLORES GORDÓN, M. (eds.). Diccionario y aprendizaje del español. Suiza. Peter Lang, 133-154.

PORTO DAPENA, J. A. (2002). Manual de técnica lexicográfica. Madrid. Arco/Libros, S.A.

PRADO ARAGONÉS, J. (2004). El ejemplo lexicográfico como referente cultural en la enseñanza de español como lengua extranjera. En GALLOSO CAMACHO, M. V. y PRADO ARAGONÉS, J. (coord.). Diccionario, léxico y cultura. Universidad de Huelva, 157-173.

RODRÍGUEZ ORTIZ, F. (2012). Ilustraciones de la técnica en la lexicografía española. Revista de lexicografía, vol. xviii, 171-187.

RODRÍGUEZ ORTIZ, F. y GARRIGA ESCRIBANO, C. (2016). Ilustraciones de la ciencia en la lexicografía española. En DE BENI, M. (eds.). Ciencias y traducción en el mundo hispánico. Mantua. Universitas Studiorum Editrice, 301-331.

RUHSTALLER, S. (2010). El léxico formal en los diccionarios monolingües de aprendizaje del español como L2. En RUHSTALLER, S y DOLORES GORDÓN, M. (eds.). Diccionario y aprendizaje del español. Suiza. Peter Lang, 233-253. 
SÁNCHEZ LÓPEZ, I. (2020). Pasado, presente y futuro de los diccionarios para el aprendizaje del español. En Monográficos SINOELE. Shanghái, 432-444.

TARP, S. (2006). Lexicografía de aprendizaje. Cadernos de Traduçao, 2(18), 295-317.

TARP, S. (2011). Pedagogical lexicography: Towards a new and strict typology corresponding to the present state-of-the-art. Lexikos, 21, 217-231. 


\section{Apéndice}

anunciar v. 1 Hacer saber, proclamar, avisar o publicar: En la radio han anunciado lluvias para los próximos días. 2 Hacer o dar publicidad con fines comerciales: Las grandes compañías comerciales anuncian sus productos en la prensa, la radio y la televisión. 3 Referido a algo que sucederá en el futuro, dar señal o indicio de ello; pronosticar: Las nubes anuncian lluvia. 4 Referido a una persona, hacer saber su nombre a otra: El mayordomo nos anunció al dueño de la casa. $\square$ ETIMOL. Del latín annuntiare. $\square$ ORTOGR. La $i$ nunca lleva tilde. (Clave)

anunciar v. 1 Hacer saber, proclamar, avisar o publicar: En la radio han anunciado lluvias 2 Hacer o dar publicidad con fines comerciales: Las grandes compañías comerciales anuncian sus productos en los medios de comunicación. 3 Referido a algo que sucederá en el futuro, dar señal o indicio de ello; pronosticar: Las nubes anuncian lluvia. $\square$ SIN. pronosticar. 4 Referido a una persona, hacer saber su nombre a otra: El mayordomo nos anunció al dueño de la casa. $\square$ La $i$ nunca lleva tilde. $\square$ Conjug. $\rightarrow$ HABLAR (4). $\square$ FAM. anunciador(dora), anunciante, anunciable. (DELE)

\begin{tabular}{|l|c|c|r|r|r|c|}
\hline & Acepciones & Definiciones & Ejemplos & $\begin{array}{l}\text { Marcas de } \\
\text { uso }\end{array}$ & Etimología & Pronunciación \\
\hline Clave & 4 & $\mathrm{M}$ & $4 / 2,3$ & 0 & 1 & 0 \\
\hline DELE & 4 & $\mathrm{M}$ & $4 / 2,3$ & 0 & 0 & 0 \\
\hline & Ortografía & Morfología & Semántica & Sintaxis & Uso & $\begin{array}{l}\text { Sinónimo/ } \\
\text { antónimo }\end{array}$ \\
\hline Clave & 1 & 0 & 0 & 0 & 0 & 1 \\
\hline DELE & 1 & 1 & 0 & 0 & 0 & 1 \\
\hline
\end{tabular}

Notas: 1. En la casilla que corresponde a acepciones y ejemplos se apunta el número de acepciones y ejemplos. Si existe alguna diferencia, se anota el número de serie, separado con una barra. 2. En la casilla que corresponde a definiciones, si son las mismas, se apunta la letra $M$; si existe alguna diferencia, se anota el número de serie. 3. En la casilla que corresponde al resto de los parámetros, si en el diccionario se observa dicha información, se apunta el 1 ; si no, se marca el 0 . 